\title{
Synaptic Channel Model Including Effects of Spike Width Variation
}

\author{
Hamideh Ramezani \\ Next-generation and Wireless Communications \\ Laboratory (NWCL) \\ Department of Electrical and Electronics \\ Engineering \\ Koc University, Istanbul, Turkey \\ hramezani13@ku.edu.tr
}

\begin{abstract}
An accurate model for neuro-spike communication is important in understanding the fundamentals of molecular communication. However, none of the existing models in the literature studied variations in the shape of action potentials during Axonal propagation, one of the steps during neurospike communication. These variations affect the amount of information communicated through a neuron. Hence, analyzing effects of these variations in the release of neurotransmitter, the carrier of information in neuro-spike communication, is imperative in deriving a realistic model for neuro-spike communication. In this work, we improve the existing channel models for synaptic communication to cover the effect of changes in the width of action potential on hippocampal pyramidal neurons based on the experimental data reported in the literature. The receiver neuron is assumed to detect spikes based on Neyman-Pearson method. We derive the structure of this detector for the proposed channel model. Numerical results depict that an increase in the spike width decreases the error probability.
\end{abstract}

\section{Categories and Subject Descriptors}

K.5.1 [Modeling and Simulation]: Model development and analysis; K.5.3 [Modeling and Simulation]: Simulation types and techniques-Molecular simulation; L.3.9 [Physical sciences and engineering]: Electronics

\section{Keywords}

Nanoscale communication, Neuro-spike communications, Vesicle release, Synaptic channel

\section{INTRODUCTION}

Nanomachines are able to do tasks such as computing, data storing, sensing and actuation. However, as a result of their size, they need to establish networks with each other

Permission to make digital or hard copies of all or part of this work for personal or classroom use is granted without fee provided that copies are not made or distributed for profit or commercial advantage and that copies bear this notice and the full citation on the first page. Copyrights for components of this work owned by others than ACM must be honored. Abstracting with credit is permitted. To copy otherwise, or republish, to post on servers or to redistribute to lists, requires prior specific permission and/or a fee. Request permissions from Permissions@acm.org.

NANOCOM '15, September 21 - 22, 2015, Boston, MA, USA

(C) 2015 ACM. ISBN 978-1-4503-3674-1/15/09 ...\$15.00.

DOI: http://dx.doi.org/10.1145/2800795.2800811.

\author{
Ozgur B. Akan \\ Next-generation and Wireless Communications \\ Laboratory (NWCL) \\ Department of Electrical and Electronics \\ Engineering \\ Koc University, Istanbul, Turkey \\ akan@ku.edu.tr
}

to become capable for more complex tasks. The resulting nanonetwork is applicable for a wide range of areas such as

- Biomedical, which includes drug delivery systems, health monitoring, genetic engineering, and immune system support.

- Industrial, which includes development of nanorobots, nanoprocessors and nanomemory

- Environmental applications such as water and air pollution control, biodegeneration, and animals and biodiversity control [1].

Among the proposed paradigms for nanonetworks, molecular communication in which molecules are used to encode, transmit and receive information is the most promising paradigm. The main reason is the fact that molecular communication exists in the structure of any living organism and is a biocompatible and biostable solution [1]. One of the significant mechanisms for molecular communication inside the human body is the ultra-large scale network of nerve cells, i.e., neurons, which is called as nanoscale neuro-spike communication [3].

Neuro-spike communication contains three phases: axonal propagation, synaptic propagation, and spike generation [3]. As depicted in Fig. 1, three main parts of each neuron participate in this communication: dendrite, soma, and axon. In the spike generation phase, neurons receive stimulation from their dendrites. If the received stimulation is strong enough, then the neuron will fire a molecular impulse signal known as action potential or spike. Then, in the axonal propagation phase, these spikes pass through the axon until they reach the axonal terminals. Axonal terminals are the places where a neuron communicates with other cells. A gap, which is called synapse, exists between the sender neuron and the receiver neuron. The arrival of a spike to the axonal terminal moves the neuron to the synaptic propagation phase. In this phase, vesicles, groups of chemical substances known as neurotransmitters, are released to the synapse. Neurotransmitters diffuse through the synapse. Some of them stimulate the receiver neuron, known as postsynaptic neuron, by binding to its receptors. Others leave the synapse, re-uptake or diffuse to other synapses and bind with other neurons.

Proposing a proper model for neuro-spike communication is a progressive step in finding a model for molecular communication. The existing models in the literature for neuro- 


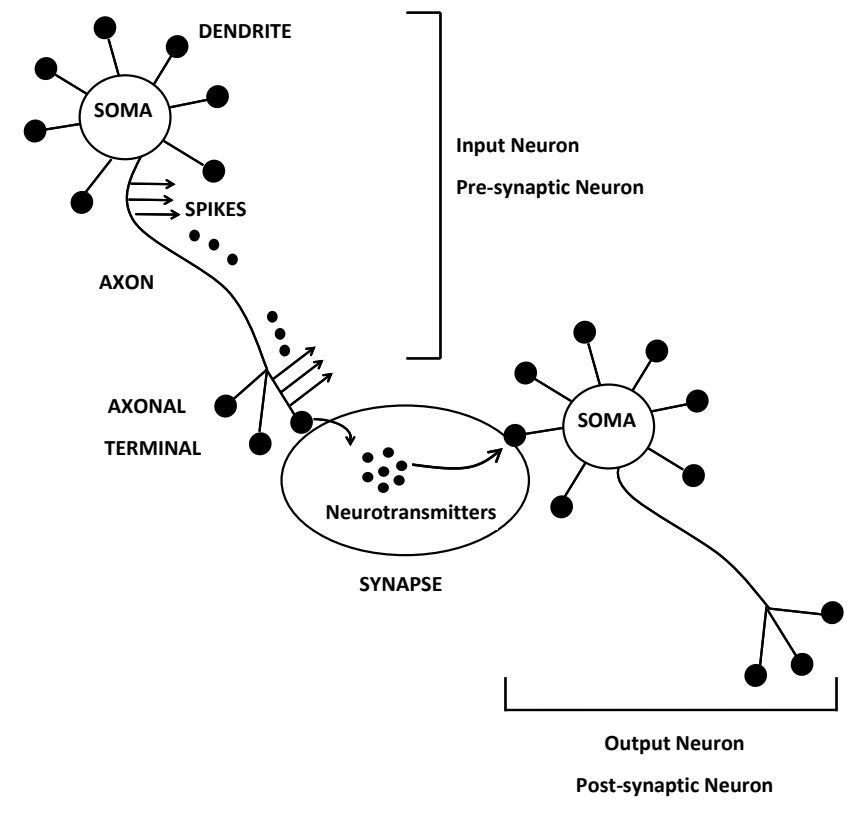

Figure 1: Neuro-spike communication.

spike communication focus on modeling synaptic communication $[3,10,11]$. However, none of these work studies variation in the shape of action potential, which has recently been recorded with the use of direct recording from axons $[6,8$, $15,18]$. These variations affect the process of the release of neurotransmitters to the synapse $[4,8,16,18]$, which affects amount of stimulation of the target cell. Hence, to reach a proper model for synaptic communication, the effects of these variations on vesicle release should be examined.

In this work, we develop a synaptic channel model for the pyramidal neurons in Cornu Ammonis (CA) area of hippocampus location in the brain which is also considered in [10]. The proposed channel model includes the effects of variation in the width of pre-synaptic spikes. We assume that the receiver neuron will detect spikes based on NeymanPearson method and derive the structure of this receiver. At the end we examine the effects of spikes width variation on the efficiency of the system with finding the probability of error detection numerically. Simulation results depict that increasing the width of the pre-synaptic spike decreases the error probability.

The remaining parts are as follows. In Section 2, we review the neural signaling background for effects of changes in the width of spikes during axonal propagation on synaptic propagation. Then, we report the existing synaptic channel models for hippocampal pyramidal neurons. In Section 3, we modify the existing models in the literature according to the effects of spike width variation on the vesicle release. In section 4, we examine the efficiency of the proposed channel based on probability of error detection. Finally we conclude our work in Section 5.

\section{BACKGROUND}

Here, first we review the neural signaling backgrounds of effects of spike width variation on vesicle release. Then, we report on the existing synaptic channel models in the literature.

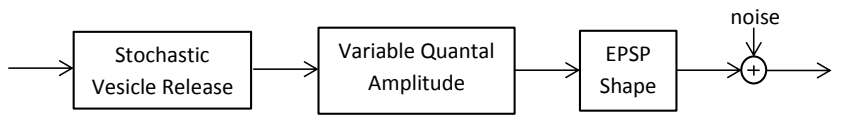

Figure 2: Structure of synaptic communication channel.

\subsection{Neural Signaling Background}

The arrival of an action potential opens voltage-dependent calcium channels (VDCC), which causes an influx of $\mathrm{Ca}^{2+}$ to the pre-synaptic terminal. A rise in $\mathrm{Ca}^{2+}$ is necessary and sufficient for releasing neurotransmitter [5]. Changes in the shape of pre-synaptic action potential modulate the concentration of $\mathrm{Ca}^{2+}$ in the terminal, which in turn, affect neurotransmitter release to the synapse $[13,18]$.

Based on results of [13], increasing the width of the action potential shifts the curve of the neurotransmitter release to the synapse. Thus, in order to derive an accurate synaptic channel model, we should find parameters which are related to the spike width and apply effects of spike width variations on these parameters. In this work we propose the first synaptic channel model which includes effects of spike width variations.

\subsection{Related Works}

Fig. 2 contains the structure of the existing models in the literature for synaptic communication channel $[3,10$, 11]. The first block is used to model stochastic release of vesicles upon arrival of spikes. The second and third blocks are used to model excitatory post synaptic potential (EPSP) generation in the post-synaptic neuron. Each of these steps are explained in the following.

\subsubsection{Vesicle Release}

A pool based model is used in the literature to model the vesicle release procedure. Based on the availability of vesicles for release, they can be grouped to 3 different pools: the readily releasable pool (RRP), the recycling pool(RP), and the reserve pool. All vesicles in RRP and RP are at the total recycling pool (TRP). In a resting synapse, only a small percentage of vesicles are ready to release. These vesicles belong to the RRP [14]. Fig. 3 contains the pool based model of the vesicle release $[2,14]$. Where $\mathrm{N}$ is size of RRP, i.e., the number of vesicles in this pool, $P_{r}(N)$ is probability of vesicle release, and $\tau_{D}$ is the time constant of recovering the TRP. Here, $N_{R}$ is the size of reserve pool and $N_{A}$ is the size of TRP.

The release of a single vesicle as a result of incoming a spike occurs according to the Poisson process with rate $\lambda_{v}(t)$, which is significant only during the arrival of presynaptic pulse. Thus, the fusion rate of a single vesicle is $\alpha_{v}=\int \lambda_{v}(t) d t$ over duration of pre-synaptic spike [12].

Two versions of the model of release terminal, which is called active zone, exist in the literature [12]:

- Active zones with univesicular release: In this model, the release of one vesicle prevents the release of others. Thus, the probability of releasing one vesicle to the synapse is one minus the probability of failure. $N$ vesicles exist in the RRP, with failure probability $\exp \left(-\alpha_{v}\right)$. Hence, the failure probability is $\exp \left(-N \alpha_{v}\right)$ and the probability of release of one vesicle upon arrival of the pulse is $P_{r}(N)=1-\exp \left(-N \alpha_{v}\right)$. 


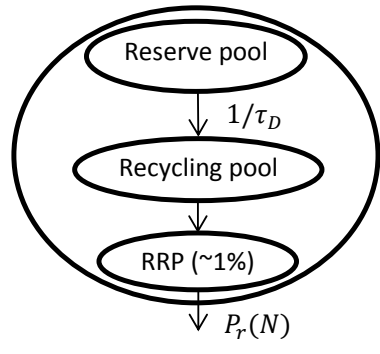

Figure 3: Pool based model of vesicle release.

- Active zones with unconstraint release: In this model, different vesicles release independently with the probability $p_{v}=1-\exp \left(-\alpha_{v}\right)$. The number of released vesicles upon arrival of a pulse, $\Delta N$, is determined based on binomial distribution with $p_{v}$ and $N$. In this case, the amplitude of the post-synaptic response depends on the numbers of bound receptors.

\subsubsection{Variable Quantal Amplitude}

To model the trial to trial variability in the amplitude of the synaptic response, the amplitude of the response is multiplied with a random variable $q$ with probability distribution of $P(q)$ [11]. $P(q)$ is modeled by a gamma distribution in the literature [10] as (1)

$$
f(q ; \alpha, \beta)=\beta^{\alpha} \frac{1}{\Gamma(\alpha)} q^{\alpha-1} \exp (-\beta q), q \geq 0, \alpha, \beta>0,
$$

where $\alpha$ is the order of gamma distribution and $\Gamma(\alpha)$ is the gamma function and is calculated based on (2). We select $\alpha=\beta=0.6^{-1}$ the same as $[10]$.

$$
\Gamma(\alpha)=\int_{0}^{\infty} x^{\alpha-1} e^{-x} d x
$$

\subsubsection{EPSP Shape}

Three kinds of receptors exist in these post-synaptic neurons: mGluRs, N-Methyl-D-Aspartate (NMDA), and AlphaAmino-3-Hydroxy-5-Methyl-4-Isoxazole-propionate (AMPA). The normalized response of all of these receptors to a neurotransmitter is modeled by an alpha function [3] as

$$
\alpha(t)=\frac{t}{\tau} \exp \left(1-\frac{t}{\tau}\right) u(t) .
$$

Where $\tau$ is a constant related to the type of the receptor. Assuming $\tau_{1}, \tau_{2}$, and $\tau_{3}$ for AMPA, NMDA, and mGluRs receptors respectively with $\tau_{3} \gg \tau_{2}>\tau_{1}$.

\subsubsection{Noise}

Post-synaptic membrane voltage is corrupted due to different noise sources like thermal noise, channel noise and synaptic noise. Synaptic noise is due to multiple accesses to synapse from thousands of other neurons. Since the signal of different neurons has the same random structure and due to the central limit theorem, the probability density function of the post-synaptic noise converges to a Gaussian process $[3,11]$.

\section{MODEL DESCRIPTION}

The existing models in the literature for synaptic communication defined spikes as all or none. However the width

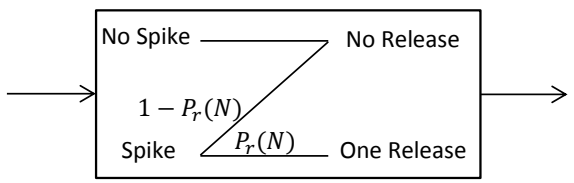

Figure 4: Vesicle release for each terminal.

of spikes may vary during axonal transmission according to history of neuron from previous stimuli. These variations in the spike width affect the release of vesicles to the synapse. In this section, we derive a synaptic communication model which contains the effects of these variations.

Based on [2], release terminals in the hippocampal pyramidal neurons have a single active zone and only one vesicle can release per action potential from each active zone. Hence, with use of univesicular model for active zones, vesicle release from each terminal is modeled with a binary $\mathrm{Z}$ channel as depicted in Fig. 4. Note that the the probability of spontaneous release, i.e, release of a vesicle without arrival of a spike, from these neurons is very low [10], and hence, we ignore it in the channel model.

Based on [18], the variation of charge transfer through VDCC has a linear relation with changes in width of action potential and is modeled as

$$
\frac{R_{C a}}{R_{C a, C}}=0.6 \frac{w}{w_{C}}+0.5 .
$$

where $w$ and $R_{C a}$ are width of spike and release of $C a^{2+}$. A neuron which is not stimulated during last seconds is in control situation and width of its response to stimulus is roughly constant. Here $(.)_{C}$ is amount of the desired parameter when the neuron is in the control situation. The relation between a change in pre-synaptic $\mathrm{Ca}^{2+}$ influx and neurotransmitter release is modeled with a power function [13] as

$$
\frac{R_{N t}}{R_{N t, C}}=0.68\left(\frac{R_{C a}}{R_{C a, C}}\right)^{0.94}+0.59 .
$$

where $R_{N t}$ is release of neurotransmitter.

In calculation of fusion rate, we should take integral of $\lambda_{v}(t)$ over width of spike. However, time domain should be discretized in the simulations. In the existing models in the literature, dividing the time domain to equal length windows with a length equal to a spike width is suggested so that there is at most one spike in each window [10]. However, we know that spike width can increase to three time of its value in control situation [8], hence we cannot select a fixed window length. One solution can be selecting windows with different lengths according to spike width, instead, we can simply consider equal length windows and take the integral of $\lambda_{v}(t)$ over one window, then update fusion rate, i.e.,

$$
\frac{\alpha_{v}}{\alpha_{v, C}}=0.68\left(0.6 \frac{w}{w_{C}}+0.5\right)^{0.94}+0.59 \text {. }
$$

Each neuron could make multiple synapses with a post synaptic neuron [17]. Assume that the number of synapses is equal to $t$ and size of RRP of $i^{t h}$ pre-synaptic terminal is equal to $N_{i}$, the probability of vesicle release from $i^{t h}$ pre-synaptic terminal is

$$
P_{r}\left(N_{i}\right)=1-\exp \left(-N_{i} \alpha_{v, i}\right) .
$$

where $\alpha_{v, i}$ is the fusion rate of $i^{t h}$ pre-synaptic terminal. 
The vesicle release from different terminals is assumed to be independent. Define all possible combinations of selecting $k$ terminals out of $t$ as members of set $C$, i.e, each member of this set is in the form of $A_{j}=\left\{j_{1}, j_{2}, \ldots, j_{k}\right\}$ where $j_{1}, j_{2}, \ldots$, and $j_{k}$ are $k$ different numbers between 1 and $t$. $A_{j}^{c}$ contains all numbers between 1 and $t$ except members of $A_{j}$. Then, the probability of releasing $k$ vesicles from $t$ terminals can be calculated as

$$
\operatorname{Pr}(k \text { release })=\sum_{A_{j} \in C}\left[\prod_{m \in A_{j}} \operatorname{Pr}\left(N_{m}\right) \prod_{l \in A_{j}^{c}}\left(1-\operatorname{Pr}\left(N_{l}\right)\right)\right] .
$$

by inserting (7) in (8), we obtain

$$
\begin{gathered}
\operatorname{Pr}(k \text { release })=\sum_{A_{j} \in C}\left[\prod_{m \in A_{j}}\left(1-\exp \left(-N_{m} \alpha_{v, m}\right)\right)\right. \\
\left.\exp \left(-\sum_{l \in A_{j}^{c}} N_{l} \alpha_{v, l}\right)\right] .
\end{gathered}
$$

However, (9) contains $\frac{t !}{k !(t-k) !}$ terms and is hard to calculate. Let $x_{i}$ be a random variable with the Bernoulli distribution as

$$
x_{i}=\left\{\begin{array}{ll}
0, & 1-\operatorname{Pr}\left(N_{i}\right) \\
1, & \operatorname{Pr}\left(N_{i}\right)
\end{array} .\right.
$$

where $x_{i}$ shows the number of released vesicles by the $i^{t h}$ terminal. Release of $k$ vesicles from $t$ terminals is the same as $\sum_{i=1}^{t} x_{i}=k$, which can be modeled by Poisson Binomial distribution with mean, $\mu$, and variance, $\sigma^{2}$, as

$$
\begin{gathered}
\mu=\sum_{i=1}^{t} P_{r}\left(N_{i}\right) \\
\sigma^{2}=\sum_{i=1}^{t} P_{r}\left(N_{i}\right)\left(1-P_{r}\left(N_{i}\right)\right) .
\end{gathered}
$$

Thus, the probability of releasing $k$ vesicles is calculated based on [7];

$$
\operatorname{Pr}(k \text { release })=\frac{1}{t+1} \sum_{l=0}^{t} D^{-l k} \prod_{m=1}^{t}\left[1+\left(D^{l}-1\right) \operatorname{Pr}\left(N_{m}\right)\right]
$$

where $D=\exp \left(\frac{2 i \pi}{t+1}\right)$ and $i=\sqrt{-1}$.

Assuming that after each vesicle release a fixed number of neurotransmitters, $N_{N t}$, bind with post-synaptic receptors. Hence, number of bound neurotransmitters is $j=k N_{N t}$ totally. Because the response of AMPA and NMDA receptors are dominant in these neurons, we neglect $\mathrm{mGluRs}$ receptors [3]. Fig. 5 shows the structure of synaptic communication with $a$ AMPA and $(j-a)$ NMDA receptors, where $0 \leq a \leq j$. $S(t)$ is the input to pre-synaptic terminals which contains spikes with different width. $\operatorname{Pr}$ (Release) is probability of releasing vesicles upon arrival of a spike and is

$$
\operatorname{Pr}(\text { Release })=\sum_{k=1}^{t} \operatorname{Pr}(k \text { release }) \text {. }
$$

$W_{i}(t)$ for $1 \leq i \leq j$ is the zero mean synaptic noise for different terminals. However different synaptic terminals have different distances from cell body, in hippocampal pyramidal neurons, EPSP amplitude is independent from distance between the synapse and the cell body [9]. Hence, the structure of receiver is the same for all AMPA and NMDA receptors in our model.

As depicted in Fig. 5, we use the optimum receiver for spike generation [3]. Based on optimum receiver, two hypotheses exist for detection, $H_{0}$ when there is no spike in the input and $H_{1}$ when there is a spike in $S(t)$. These hypotheses are

$$
\begin{gathered}
H_{1}: v=\sum_{i=1}^{j} q_{i} c_{i}+n_{i} \\
H_{0}: v=\sum_{i=1}^{j} n_{i}
\end{gathered}
$$

where $q_{i}$ is the variable quantal amplitude for $i^{\text {th }}$ received signal, $c_{i}$ and $n_{i}$ are

$$
\begin{aligned}
& c_{i}=\int_{T_{n}}^{T_{n+1}} \alpha_{A}^{2}(\tau) d \tau=c_{A} \\
& n_{i}=\int_{T_{n}}^{T_{n+1}} W_{i}(\tau) \alpha_{A}(\tau) d \tau
\end{aligned}
$$

for $1 \leq i \leq a$ and

$$
\begin{aligned}
& c_{i}=\int_{T_{n}}^{T_{n+1}} \alpha_{N}^{2}\left(\tau-t_{0}\right) d \tau=c_{N} \\
& n_{i}=\int_{T_{n}}^{T_{n+1}} W_{i}(\tau) \alpha_{N}\left(\tau-t_{0}\right) d \tau
\end{aligned}
$$

for $a+1 \leq i \leq j$ respectively. Where $\left[T_{n}, T_{n+1}\right]$ is the range of the desired window, $\alpha_{A}(t)$ is the response of AMPA receptors, $\alpha_{N}\left(t-t_{0}\right)$ is the response of the NMDA receptors to a neurotransmitter binds to them at time $t$, and $t_{0}>0$ is the delay of NMDA receptor. In [3] the threshold value for decision is found based on Neyman-Pearson method by

$$
\text { Threshold }=\ln \Omega-\ln \frac{\sigma_{0}}{\sigma_{1}}+\frac{\mu_{1}^{2}}{2 \sigma_{1}^{2}} .
$$

where $\Omega, \mu_{1}, \sigma_{0}$, and $\sigma_{1}$ are the ratio of probability of receiving $v$ when there is a spike and when there is no spike, mean of $v$ when there is a spike, variance of $v$ when there is no spike, and variance of $v$ when there is a spike respectively. These parameters are calculated as

$$
\begin{gathered}
\Omega=\frac{\frac{1}{\sqrt{2 \pi \sigma_{1}^{2}}} \exp \left(-\frac{\left(v-\mu_{1}\right)^{2}}{2 \sigma_{1}^{2}}\right)}{\frac{1}{\sqrt{2 \pi \sigma_{0}^{2}}} \exp \left(-\frac{v^{2}}{2 \sigma_{0}^{2}}\right)} \\
\mu_{1}=E\left[v \mid H_{1}\right]=E\left[\sum_{i=1}^{j} c_{i} E\left[q_{i}\right]\right] \\
\sigma_{0}=\operatorname{Var}\left[v \mid H_{0}\right]=E\left[\sum_{i=1}^{j} \operatorname{Var}\left[n_{i}\right]\right] \\
\sigma_{1}=\operatorname{Var}\left[v \mid H_{1}\right]=E\left[\sum_{i=1}^{j} c_{i}^{2} \operatorname{Var}\left[q_{i}\right]+\operatorname{Var}\left[n_{i}\right]\right]+ \\
\operatorname{Var}\left[\sum_{i=1}^{j} c_{i} E\left[q_{i}\right]\right] .
\end{gathered}
$$




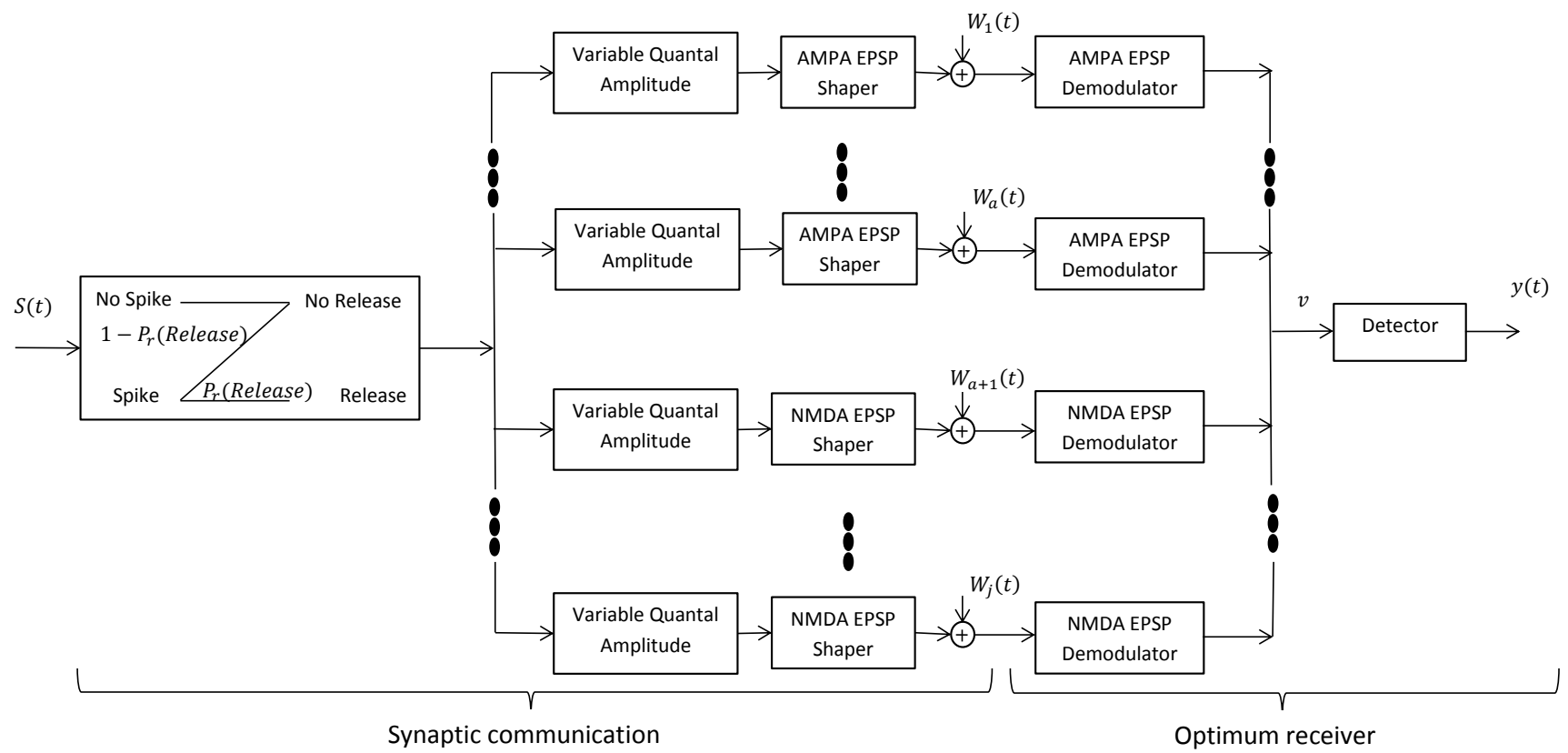

Figure 5: Synaptic communication with optimum receiver for spike generation.

Define the number of AMPA receptors, $a$, as a fixed fraction of all bound neurotransmitters, i.e., $a=f j$ and $0 \leq f \leq 1$. By assuming iid channels with iid noises, (23), (24), and (25) will simplify to

$$
\begin{gathered}
\mu_{1}=\left(f c_{A}+(1-f) c_{N}\right) E[q] E[j] \\
\sigma_{0}=E[j] \operatorname{Var}[n] \\
\sigma_{1}=\left(f c_{A}^{2}+(1-f) c_{N}^{2}\right) E[j] \operatorname{Var}[q]+E[j] \operatorname{Var}[n]+ \\
\left(f c_{A}+(1-f) c_{N}\right)^{2} E[q]^{2} \operatorname{Var}[j] .
\end{gathered}
$$

where $E\left[q_{i}\right]=E[q], \operatorname{Var}\left[q_{i}\right]=\operatorname{Var}[q]$, and $\operatorname{Var}\left[n_{i}\right]=\operatorname{Var}[n]$ for $1 \leq i \leq j$. Mean and variance of $j$ are calculated as

$$
E[j]=N_{N t} E[k]=N_{N t} \sum_{i=1}^{t} P_{r}\left(N_{i}\right)
$$

$$
\operatorname{Var}[j]=N_{N t}^{2} \operatorname{Var}[k]=N_{N t}^{2} \sum_{i=1}^{t} P_{r}\left(N_{i}\right)\left(1-P_{r}\left(N_{i}\right)\right) .
$$

\section{RESULTS}

To find effects of spike width variations on vesicle release, we find the error probability of the system for different spike width. Parameters of the model are selected as Table 1 based on $[3,10]$.

Error probability for different amount of $t$ and spike width is plotted in Fig. 6. We observe that increasing the width of spike decreases the error probability as it increases $\alpha_{v}$ based on (6) which increases vesicle release probability for all terminals. Furthermore, based on Fig. 6, increasing $t$ also decrease error probability. The reason is that increasing $t$ will increase probability of having more bound neurotransmitters to the post-synaptic terminal.

Note that error probability of the channel by neglecting effects of spike width variations is the same as plotted error probability with normalized width equal to 1 . Thus,

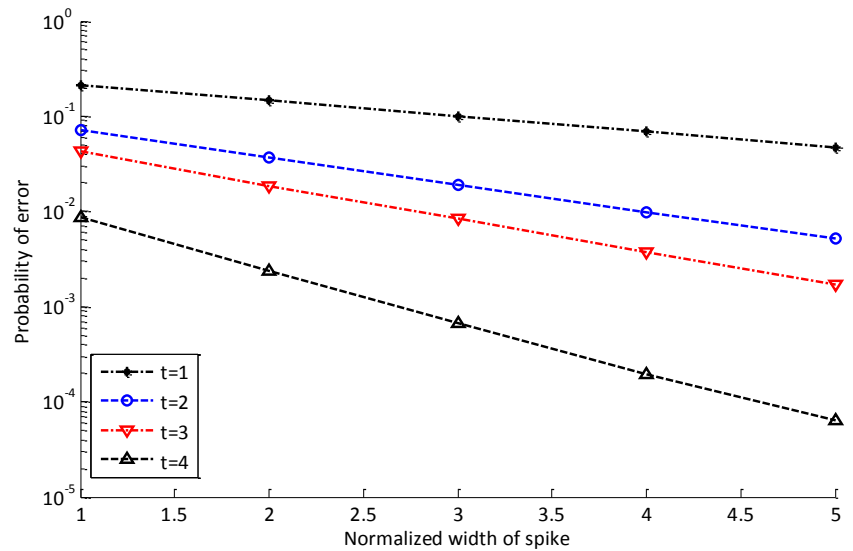

Figure 6: Error probability under different number of synaptic terminals and spike widths.

we see that the increase in the spike width improves the efficiency of the synaptic channel which will affect the efficiency of the neuro-spike communication. Hence, a proper model for neuro-spike communication should contain effects of changes in the shape of the spike during axonal transmission on synaptic propagation and spike generation phases.

\section{CONCLUSIONS}

In this work we proposed the first synaptic communication channel model which includes effects of spike width variation. In our model, we considered existing of multiple terminals with univesicular release, which is realistic for neurons in Hippocampal CA region, between two neurons. Different terminals have different release probabilities. Hence, we modeled number of released vesicles with Poisson Binomial 
Table 1: Simulation parameters for synaptic channel model

\begin{tabular}{|l|l|l|}
\hline Parameter & Symbol & Value \\
\hline Time constant of AMPA and NMDA receptors & $\tau_{1}$ and $\tau_{2}$ & 8 and $10(\mathrm{~ms})$ \\
\hline Start time of $n^{t h}$ and $(n+1)^{t h}$ window & $T_{n}$ and $T_{n+1}$ & 0 and $100(\mathrm{~ms})$ \\
\hline Mean and Variance of quantal amplitude & $E[q]$ and $\operatorname{Var}[q]$ & 1 and $0.6^{2}$ \\
\hline Variance of noise in detection process & $\operatorname{Var}[n]$ & 0.01 \\
\hline Number of vesicles in $i^{t h}$ terminal & $N_{i}$ & Uniformly distributed between $[3-5]$ \\
\hline Number of bound neurotransmitters per vesicle & $N_{N t}$ & 11 \\
\hline Fusion rate in control situation & $\alpha_{v}, C$ & 2 spikes $/ s$ \\
\hline Fraction of AMPA receptors & $f$ & 0.7 \\
\hline Probability of existing a spike in $S(t)$ & & 0.8 \\
\hline
\end{tabular}

distribution. Simulation results showed that an increase in spike width, which may happen according to history of neurons, decreases the probability of error detection.

\section{ACKNOWLEDGMENTS}

This work was partly supported by the European Research Council (ERC) under grant ERC-2013-CoG \#616922, and by Turkish Scientific and Technical Research Council under grants \#109E257 and BIDEB-2215.

\section{REFERENCES}

[1] I. F. Akyildiz, F. Brunetti, and C. Blázquez. Nanonetworks: A new communication paradigm. Comput. Netw., 52(12):2260-2279, Aug. 2008.

[2] A. A. Alabi and R. W. Tsien. Synaptic vesicle pools and dynamics. Cold Spring Harbor Perspectives in Biology, 4:a013680, 2012.

[3] E. Balevi and O. Akan. A physical channel model for nanoscale neuro-spike communications. Communications, IEEE Transactions on, 61(3):1178-1187, March 2013.

[4] S. Debanne and et al. What are the mechanisms for analogue and digital signalling in the brain? Nature Reviews Neuroscience, 14:63-69, 2013.

[5] K. R. Delaney and E. E. Stanley. Calcium and Neurotransmitter Release. John Wiley \& Sons, Ltd, 2001.

[6] Z. Feng, Y. Yu, Z. Guo, J. Cao, and D. M. Durand. High frequency stimulation extends the refractory period and generates axonal block in the rat hippocampus. Brain Stimulation, 7(5):680 - 689, 2014.

[7] M. Fernandez and S. Williams. Closed-form expression for the poisson-binomial probability density function. Aerospace and Electronic Systems, IEEE Transactions on, 46(2):803-817, April 2010.

[8] J. R. Geiger and P. Jonas. Dynamic control of presynaptic ca $2+$ inflow by fast-inactivating $\mathrm{k}+$ channels in hippocampal mossy fiber boutons. Neuron, 28:927-939, 2000.

[9] J. C. Magee and E. P. Cook. Somatic EPSP amplitude is independent of synapse location in hippocampal pyramidal neurons. Nature Neuroscience, 3:895-903, 2000.

[10] D. Malak and O. Akan. A communication theoretical analysis of synaptic multiple-access channel in hippocampal-cortical neurons. Communications, IEEE Transactions on, 61(6):2457-2467, June 2013.
[11] A. Manwani and C. Koch. Detecting and estimating signals over noisy and unreliable synapses: Information-theoretic analysis. Neural Comput., 13(1):1-33, Jan. 2001.

[12] V. Matveev and X. Wang. Implications of all-or-none synaptic transmission and short-term depression beyond vesicle depletion: A computational study. The Journal of Neuroscience, 20:1575-1588, 2000.

[13] J. Qian and P. Saggau. Modulation of transmitter release by action potential duration at the hippocampal ca3-ca1 synapse. Journal of Neurophysiology, 81(1):288-298, 1999.

[14] S. O. Rizzoli and W. J. Betz. Synaptic vesicle pools. Nature Reviews Neuroscience, 6:57âĂŞ69, 2005.

[15] T. Sasaki. The axon as a unique computational unit in neurons. Neuroscience Research, 75(2):83 - 88, 2013.

[16] T. Sasaki, N. Matsuki, and Y. Ikegaya. Action-potential modulation during axonal conduction. Science, 331(6017):599-601, 2011.

[17] G. M. G. Shepherd and K. M. Harris. Three-Dimensional Structure and Composition of CA3-CA1 Axons in Rat Hippocampal Slices: Implications for Presynaptic Connectivity and Compartmentalization. The Journal of Neuroscience, 18(20):8300âĂŞ8310, October 1998.

[18] D. Wheeler and et al. Changes in action potential duration alter reliance of excitatory synaptic transmission on multiple types of ca2 + channels in rat hippocampus. The Journal of Neuroscience, 16:2226-2237, 1996. 